\title{
RESULTADOS PARA EL DESARROLLO DE UN MANUAL DE AUTOAYUDA: MANEJO DEL ESTRÉS EN FAMILIAS CON UN CASO DE CÁNCER GÁSTRICO ${ }^{1}$
}

\section{THE RESULTS OF AN INVESTIGATION TO PRODUCE A SELF-HELP MANUAL: MANAGE OF STRESS IN FAMILIES WITH A CASE OF GASTRIC CANCER}

\author{
Catalina Espinoza Ortiz* \\ Valeryn Blanco Viales** \\ Ronald Ramírez Henderson***
}

RESUMEN

Este artículo es el resultado de un trabajo investigativo donde se aborda el tema del estrés en familias con un caso de cáncer gástrico, generando una propuesta de un manual para el manejo del estrés en familias con condiciones como la antes mencionada.

A partir del análisis de los resultados obtenidos se crea el manual, el cual contiene material escrito $y$ auditivo que pretende que las familias se preparen con habilidades para el manejo del estrés y de esta manera eviten en la medida de lo posible, que la condición estresante influya negativamente en la recuperación del paciente y en el ambiente familiar.

PALABRAS CLAVE: MANUAL * ESTRÉS * FAMILIA * CÁNCER * TERAPIA RACIONAL EMOTIVO CONDUCTUAL (TREC)

El presente artículo se desprende de una investigación mayor titulada: "Propuestas de un manual de autoayuda cognitivo, conductual emocional para el manejo del estrés en familias en donde un miembro ha sido intervenido quirúrgicamente por cáncer gástrico", realizado por las autoras del presente documento como trabajo final de graduación para optar por el grado de Licenciatura en Psicología.
Fundación Omar Dengo, Costa Rica. cattortiz@yahoo.com

** Corporación PIPASA en Liberia, Costa Rica. valerynblanco@hotmail.com

*** Escuela de Psicología de la Universidad de Costa Rica. macr0607@ice.co.cr 


\title{
ABSTRACT
}

\begin{abstract}
This investigation approaches the subject of stress in families with a case of gastric cancer, gathered information related to the cognitive, behavioral and emotional aspects that must be contained in a manual of self-help, which explains how to face situations of stress in families with the mentioned characteristics. This manual has been created from the analysis of such results, and it consists of written and auditory material, which we hope, prepares families to cope with stress management in order to avoid, as much as possible, that stress becomes a negative influence in the recovery of the patient and the family atmosphere.
\end{abstract}

KEYWORDS: MANUAL * STRESS * FAMILY * CANCER * RATIONAL EMOTIVE BEHAVIOR THERAPY (REBT)

\section{INTRODUCCIÓN}

El cáncer constituye uno de los problemas de salud de mayor impacto, tanto en Costa Rica como en el mundo. En las políticas de la Organización Mundial de la Salud (OMS, 2002), se ha establecido que esta enfermedad cobra seis millones de vidas por año en el mundo. En este contexto se desarrolla una investigación con el fin de crear un manual cuyo uso sea para personas diagnosticadas con cáncer y su grupo familiar, siendo este texto de consulta desde el momento que se da la noticia del diagnóstico hasta el tiempo de recuperación y recomendaciones de prevención para los seres cercanos. Si bien es cierto, el texto es creado con miras específicas al cáncer gástrico, las autoras no excluyen su uso y utilidad para otros tipos de cáncer.

A pesar de los grandes avances de la ciencia y la tecnología médica para su diagnóstico $y$ tratamiento, la enfermedad no disminuye. En Costa Rica específicamente, el cáncer es la segunda causa de muerte, una de cada cinco personas fallecerá por esta enfermedad cada año, el cáncer de estómago se reporta como uno de los de mayor incidencia en nuestro país (Loría, 2002). Estos datos colocan al cáncer gástrico en un lugar considerable en lo que se refiere a enfermedades representativas en nuestro país.

En Costa Rica las personas con cáncer son atendidas en sus hogares, $y$ no en instituciones, gracias a la política adoptada por las Clínicas de Cuidados Paliativos a partir de los años 80, no obstante, es hasta 1996 que se pasa de un enfoque biologista a un enfoque biopsicosocial, donde el ser humano se valora de manera integral, es decir, biológica, psicológica y socialmente (Loría, 2002).

A pesar de que la idea de involucrar al núcleo familiar en el cuidado de la persona enferma pareciera ser lo más oportuno, también trae un gran desajuste dentro del hogar. La dinámica familiar se ve alterada y nuevos roles tendrán que ser asumidos por los diferentes miembros de la familia. Por lo tanto, estos nuevos eventos serán potenciales generadores de estrés, desencadenando en las personas una serie de pensamientos y conductas que afectan negativamente su estado emocional y físico (Álvarez, 1989; Bárez, M. y Fernández, 2003; Carrobles, 1996; Cascante y Salazar, 1993; Fertig, 1997; Lazarus y Folkman, 1986; Maguire, 2000), esto sin dejar de lado las dificultades que se generan luego de que la persona ha sido intervenida quirúrgicamente y en el proceso de recuperación respectivo.

Alán y León (1999), Barguil (2003), Martínez y Valiente (1994), Moix (1994), entre otros, en sus respectivas investigaciones, detectaron que los factores psicológicos, principalmente los niveles de estrés, afectan la recuperación del paciente después de la cirugía, esto debido a que el estrés activa mecanismos tanto fisiológicos como cognitivos, conductuales y emocionales contraproducentes a la recuperación.

Por lo tanto, desde esta realidad y conociendo las consecuencias que el estrés puede causar no solo en la persona intervenida quirúrgicamente, sino también en el grupo familiar 
de la misma, se dirigió la investigación hacia la ayuda en el desarrollo de estrategias para el afrontamiento del estrés dentro de hogares, en donde uno de sus miembros ha sido diagnosticado por cáncer gástrico.

\section{METODOLOGÍA}

La investigación desarrollada se ubicó dentro del marco de un estudio exploratorio, ya que no existía en el contexto costarricense un documento igual o similar al que se propuso.

\section{PARTICIPANTES}

En total participaron 26 personas en la investigación, de las cuales 16 corresponden a cinco familias participantes en donde uno de sus miembros fue intervenido quirúrgicamente por cáncer gástrico y diez son los profesionales abordados de las áreas de Psicología, Medicina, Nutrición, Enfermería y Trabajo Social, dos por cada disciplina profesional, todos trabajando directamente con esta población.

\section{INSTRUMENTOS}

\section{CUESTIONARIOS}

Se diseñaron cuestionarios para los pacientes y los familiares participantes, basados en documentos desarrollados por Barguil (2003) y Olivares y Méndez (1998). Con las modificaciones pertinentes del caso, se midieron variables como los hábitos alimenticios, procesos de comunicación familiar, relaciones sociales, síntomas de depresión, pensamientos, conductas y sentimientos ante la noticia y la experiencia de la intervención quirúrgica. Antes de su aplicación fueron sometidos a validación por grupos familiares con características similares a la población escogida y por profesionales de las áreas de medicina y psico- logía que trabajan directamente con personas gastrectomizadas.

\section{ENTREVISTAS}

La información se completó por medio de entrevistas grupales e individuales semiestructuradas, tanto a las familias como a los profesionales de las áreas mencionadas.

\section{TIPO DE ANÁLISIS}

Para realizar una propuesta del Manual de autoayuda Cognitivo Conductual Emocional para el manejo del estrés, dirigido a familias en donde uno de sus miembros ha sido intervenido quirúrgicamente por cáncer gástrico, tomando en cuenta que uno de los objetivos principales de la investigación fue determinar las características con las que debe contar este, el análisis se realizó de manera tal, que se pudieran obtener las conductas, cogniciones y sentimientos disfuncionales presentados en estas familias. Más adelante y con base en estos resultados se buscó como un gran objetivo generar el establecimiento de estilos de vida saludables, que contribuyan al mejoramiento de la persona intervenida quirúrgicamente y propicie hábitos de prevención en el grupo familiar.

\section{ANÁLISIS DE LOS RESULTADOS}

Los principales hallazgos según la información suministrada por las familias y profesionales consultados se presentan a continuación; de manera resumida para efectos de este artículo se ha adaptado el diseño original de la UVE Heurística propuesta por Bob Gowin (1988), ya que su estructura propicia el análisis de un proceso de producción de conocimiento, en este caso, orientando los resultados de la presente investigación. 


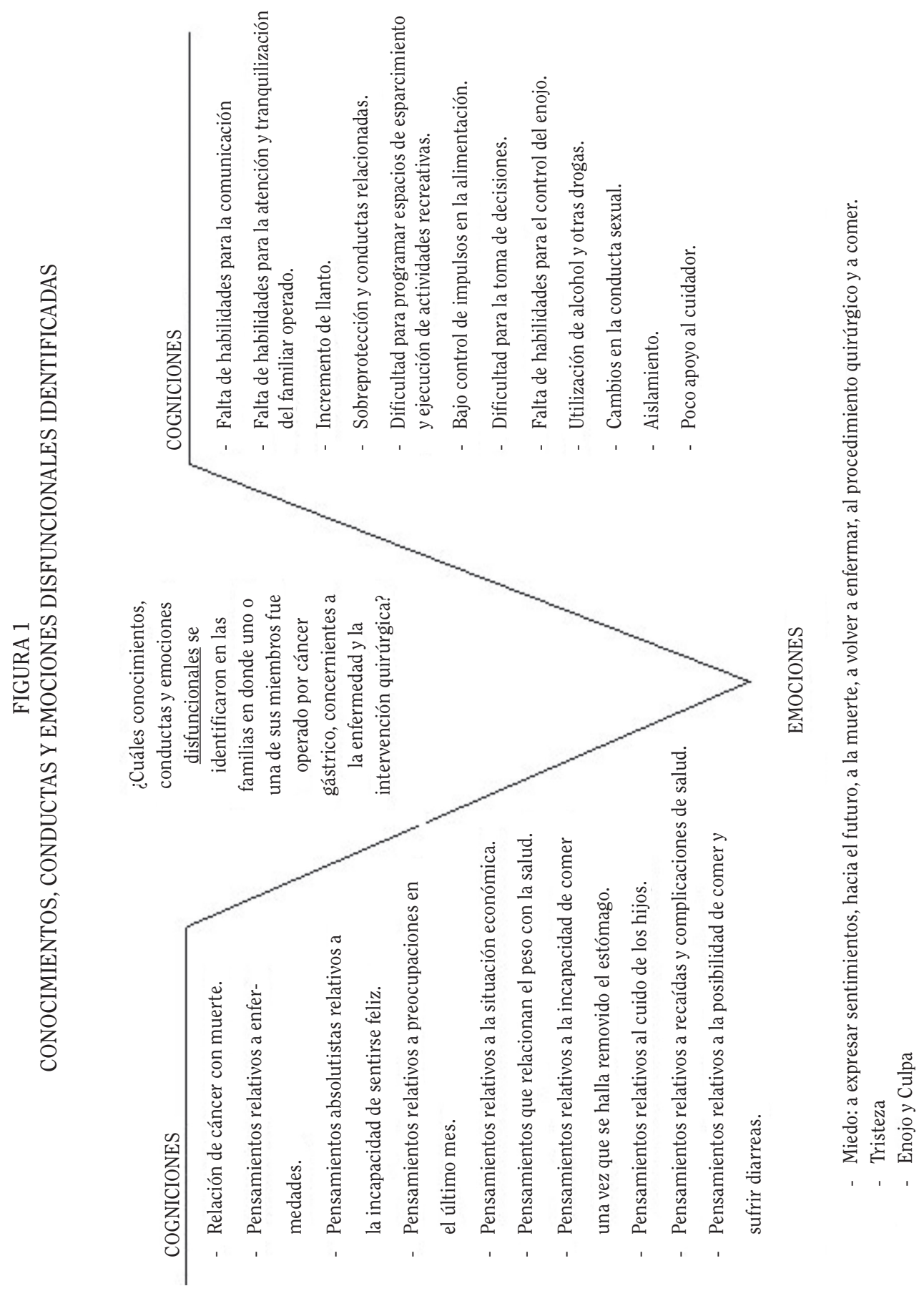




\section{ELABORACIÓN DEL MANUAL}

El documento fue elaborado según los hallazgos presentados en la figura 1. El manual se basa en preceptos teóricos revisados cuidadosamente para dar atención a las necesidades detectadas. A la vez se desarrollan tres ejes transversales a lo largo del documento, basándose la investigación en Bárez y Fernández (2003), a saber: apoyo emocional, apoyo informacional- educacional y apoyo instrumental.

Siendo el manejo del estrés la columna vertebral del manual, se brindan herramientas esenciales basándose en el Modelo Cognitivo Conductual Emocional, tomando en cuenta estrategias para comprender la relación entre pensamientos, emociones y conductas en el desarrollo de estados de estrés (Alán y León, 1999; Bayés, 1991; Ellis, 1977; Meichenbaum, 1987; Méndez y Olivares, 1998). Además de estrategias para el afrontamiento del mismo, como el control del tiempo, control del contexto y control del miedo, basándose en estudios anteriores en donde la sensación de control en estos procesos es esencial. Así mismo, se brindan técnicas para la relajación muscular y cognitiva, se hace énfasis en la importancia del ejercicio físico y la risa como estrategias complementarias para el manejo del estrés (Kirsta, A; 1986). Algunas de las técnicas mencionadas se brindan de manera audible por medio de un material complementario (disco compacto) con el objetivo de propiciar el entrenamiento en la relajación muscular y cognitiva. Para la realización de dicho material se basaron las investigadoras en los ejercicios de relajación progresiva de Jacobson (1929), así como, también en ejercicios de relajación autógena con el objetivo de estimular el lóbulo derecho del cerebro, pues cuando el hemisferio izquierdo desciende y el hemisferio derecho se convierte en dominante se genera la onda cerebral Alfa, correspondiente con estados de calma y relajación (Yanes, 2008). A continuación se presentan los principales contenidos del manual. 


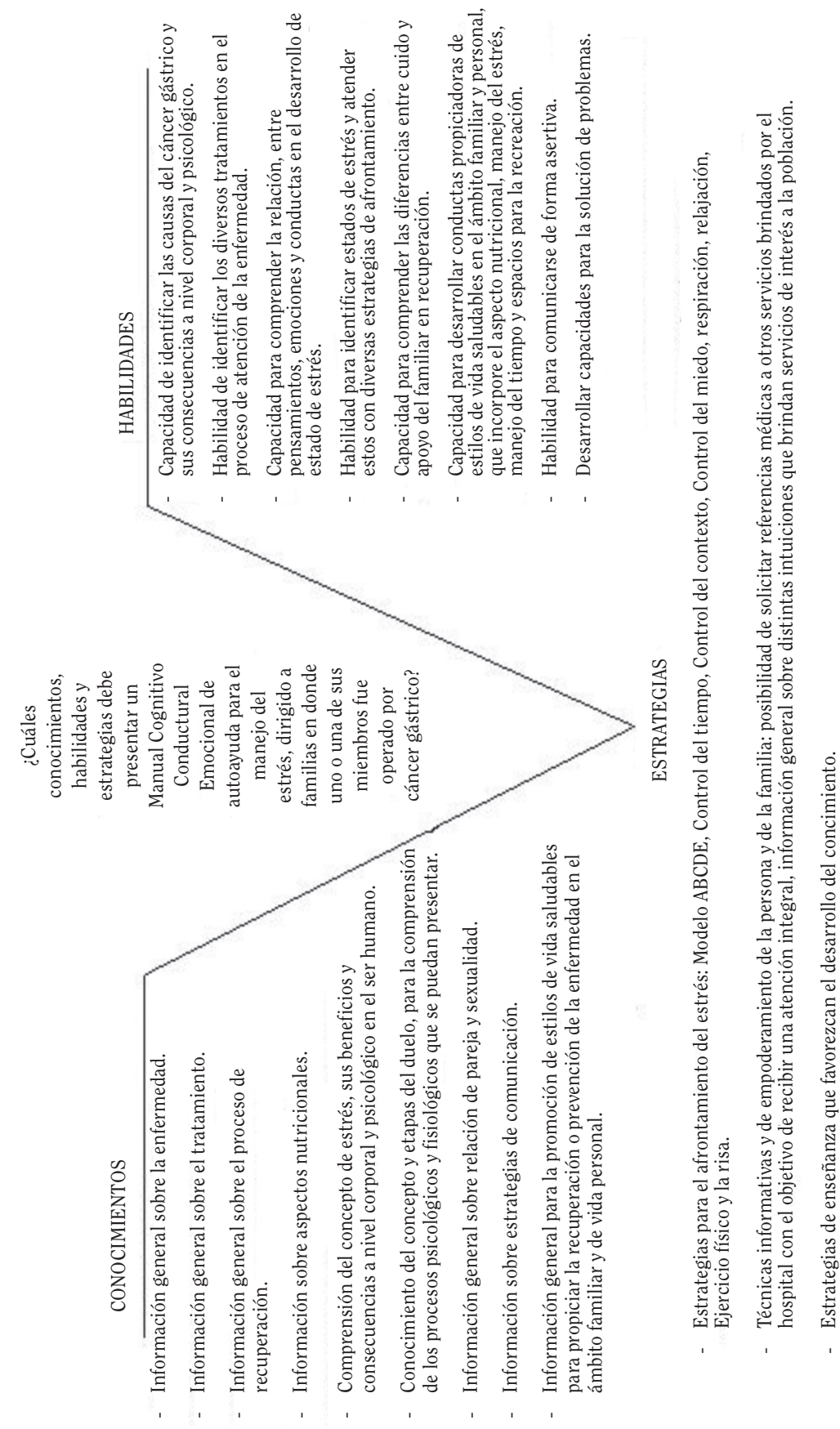




\section{DISCUSIÓN}

Con base en el interés de investigar los aspectos cognitivo, conductual y emocional necesarios para el desarrollo de un manual de autoayuda dirigido al grupo familiar en donde uno de sus miembros padece de cáncer gástrico, se mencionan los siguientes hallazgos:

\section{COGNICIONES, CONDUCTAS Y EMOCIONES DISFUNCIONALES}

Las cogniciones son evaluadas desde el género, ya que la salud y la enfermedad se vivencian de distinta manera según este (Velasco, 2006).

Para las mujeres su mayor preocupación es el cuido de los hijos. Así mismo, la investigación refleja que las conductas relacionadas con el cuido de la persona intervenida quirúrgicamente son asumidas por las mujeres, no por los hombres.

Todos los hombres intervenidos quirúrgicamente reciben el apoyo de sus esposas, no siendo este el caso de las mujeres, esto es mencionado también por los expertos consultados, en donde han detectado el poco apoyo que reciben las mujeres por parte de sus parejas, congruente además con lo encontrado en la investigación de Velasco (2006) y Chávez (1998).

Las principales conductas disfuncionales en las personas intervenidas, así como, en sus familiares son: falta de habilidades para la comunicación, para la atención y tranquilización del familiar operado, la toma de decisiones, dificultad para programar espacios de esparcimiento y ejecución de actividades recreativas, sobreprotección y bajo control de impulsos con la alimentación.

Las principales emociones disfuncionales en familiares y persona operada son: tristeza, miedo a expresar sentimientos, a la muerte, a la reincidencia de la enfermedad, incertidumbre respecto al futuro $y$ culpa. Estas emociones se desprenden de dos momentos, un primer momento, el instante de conocer el diagnóstico y la noticia de la necesidad de intervenir quirúrgicamente $y$ un segundo momento, después de la operación, que se detallan a continuación:
1. Las respuestas emocionales disfuncionales al momento de conocer la noticia del diagnóstico y su necesidad de intervenir quirúrgicamente, son el miedo a la muerte, la incertidumbre en el grupo familiar y sentimientos de incapacidad.

2. Las respuestas emocionales disfuncionales presentadas más frecuentemente en la etapa post-operatoria son el miedo a una recaída y preocupaciones económicas.

De estas cogniciones, conductas y emociones disfuncionales, se desprenden las siguientes necesidades que dan sustento a la elaboración del manual propuesto.

\section{NECESIDADES EMOCIONALES, COGNITIVAS Y CONDUCTUALES}

El manual atiende desde diferentes perspectivas, necesidades de conocimiento e información, emocionales y de conducta, con el objetivo de mejorar las relaciones del grupo familiar, así como minimizar los estados de estrés en la persona intervenida quirúrgicamente como de sus seres cercanos. Las técnicas van desde estrategias didácticas para la comprensión de la lectura, por ejemplo, preguntas sobre puntos importantes al final de cada capítulo; reforzamiento para que el grupo familiar realice las consultas necesarias por medio de espacios y cuadros, en los cuales pueden escribir las dudas que la lectura les pueda generar y las evacuen con el especialista correspondiente, hasta un CD con ejercicios de relajación muscular $y$ mental $^{2}$. La figura 2 detalla el contenido del Manual. A continuación, se detallan algunos puntos encontrados y desarrollados dentro de este:

$\diamond \quad$ El apoyo social es fundamental para contrarrestar los sentimientos de tristeza y miedo. Es necesario instrumentar en técnicas para el manejo del estrés $y$ control de emociones por medio del modelo ABCDE $y$ se hace énfasis en el

$2 \quad$ El Manual y el CD se encuentran en la biblioteca Luis Demetrio Tinoco de la Universidad de Costa Rica para su consulta pública. 
papel de la religión como canalizador social de sentimientos de esperanza, apoyo y fe.

$\diamond \quad$ Brindar mayor información a través de diversas técnicas, acerca de la enfermedad, el tratamiento, el proceso de recuperación post-operatorio, aspectos nutricionales, comprensión del concepto de estrés y del proceso de duelo, relación de pareja $y$ sexualidad, $y$ estrategias de comunicación y apoyo social, que al final brinden un conocimiento general sobre estilos de vida saludables y sus beneficios, facilitando la recuperación y promoviendo la prevención.

$\diamond \quad$ Se evidencia una necesidad de capacitación y estimulación para el desarrollo de conductas propiciadoras de estilos de vida saludables en el ámbito personal y familiar, incluyendo capacidad de comunicarse asertivamente, solucionar problemas y la administración del tiempo para realizar actividades recreativas y de ocio.

$\diamond \quad$ Siendo el miedo una de las principales emociones detectadas, el control de este se hace necesario, ya que es uno de los principales mecanismos desencadenantes de las respuestas primitivas del cerebro, como son ataque y huída, desencadenantes biológicos y naturales de los estados de estrés.

\section{TÉCNICAS PARA EL MANEJO DEL ESTRÉS}

Las investigadoras se inclinaron por el Modelo Cognitivo Conductual Emocional y dentro de este, por Técnicas Combinadas para el tratamiento del estrés, en donde se brindan entre otras, herramientas básicas en técnicas informativas, comunicación asertiva, control de impulsos y resolución de problemas.

Además, se brinda de manera auditiva entrenamiento para la relajación muscular y mental como una ayuda para el manejo del estrés. Para la realización de este CD las autoras se basaron en los ejercicios de relajación progresiva de Jacobson (1929), enfatizando el estímulo del lóbulo derecho del cerebro, que según Yanes (2008) propicia la onda cerebral Alfa correspondiente con estados de calma $y$ relajación. Cuando el hemisferio izquierdo desciende en gran medida su actividad y el hemisferio derecho alcanza la máxima, las ondas Alfa, propias del estado creativo, surgen y se generan a nivel fisiológico, estados de relajación mental.

Finalmente, la investigación deja ver la necesidad del funcionamiento de departamentos interdisciplinarios dentro de la atención pública de la salud en nuestro país, específicamente en la atención del cáncer gástrico en donde trabajen conjuntamente profesionales en el área de Psicología, Nutrición, Trabajo Social, Enfermería, Terapia Física; incluso coordinando con los departamentos de Odontología de los respectivos centros, tomando en cuenta la importancia del proceso de masticación en la recuperación de las personas intervenidas quirúrgicamente por cáncer gástrico. Una atención interdisciplinaria, propiciando estudios integrales de las familias $y$ buscando todos los recursos que el Estado puede brindarle a las personas con más necesidades, puede mejorar la calidad de vida, disminuyendo las probabilidades de contraer cáncer por parte de los otros miembros familiares, así como, favorecer la recuperación de quien ya lo padece. La idea de la creación de esta unidad o departamento dentro de la atención pública es además, que trascienda a un trabajo interinstitucional, propiciando la apertura de canales de comunicación con otras instituciones que puedan fortalecer el trabajo del personal, como por ejemplo, el IMAS y sus distintos programas de bienestar social, así como, el Instituto de Investigaciones contra el Cáncer, generando propuestas de capacitación y prevención conjuntas a la sociedad costarricense. Esto sería un medio poderoso de divulgación de estrategias de prevención de este tipo de cáncer, además de la evidente atención integral que se daría a la persona y a su familia.

La unidad propuesta podría tener además una función capacitadora y retroalimentadora al grupo de médicos. Su objetivo sería propiciar una atención integral sobre una biologista. Esta unidad se podría convertir además en un espacio catártico, de reflexión y análisis para el grupo de médicos, enfermeros y personal administrativo, recordando que son 
funcionarios que se enfrentan todos los días al desgaste emocional provocado por el trabajo con esta población.

Actualmente, considerando la realidad de la atención hospitalaria en el sector público, siendo la referencia médica, la vía más efectiva para poder accesar a la mayoría de las otras dependencias que deberían dar soporte para una atención integral (Psicología, Trabajo Social, Nutrición, Odontología) se hace necesario que los médicos estén capacitados y sumamente sensibilizados con las diferentes necesidades de las personas y sus familias para procurar una atención plena.

Además de la dieta y las recomendaciones médicas, el apoyo psicológico no debería verse como un valor agregado al tratamiento, sino como parte fundamental de este. La creación de la unidad propuesta anteriormente podría facilitar la atención psicológica de las familias que acuden a la atención pública, apoyando al grupo familiar en las primeras etapas, que como lo apunta la investigación, por el impacto de la noticia, las familias no pueden entender correctamente la información que se les está suministrando, por lo tanto, el apoyo emocional por medio de un o una profesional en el área de Psicología sería fundamental para el inicio y mantenimiento adecuado del tratamiento y las consecuentes indicaciones y cuidados del miembro intervenido quirúrgicamente. Siguiendo en esta línea, es evidente la necesidad de un trabajo conjunto entre Nutrición y Psicología, para reforzar el régimen nutricional, pero a la vez para evitar recaídas en conductas autodestructivas como comer en exceso, beber alcohol o consumir tabaco. Esto implica un cambio de hábitos y estilos de vida en muchos casos radical, muchos pacientes pueden sentir dificultad para adaptarse a las nuevas indicaciones.

Así mismo, a pesar de que el Manual se constituye de autoayuda, es importante que en estos procesos se tenga el apoyo y la atención de un profesional en Psicología que le pueda orientar y apoyar en las diferentes etapas de intervención y recuperación de la enfermedad, tanto a nivel individual como familiar.

Las necesidades sociales en nuestro país son numerosas, por lo tanto, las investigaciones que se generen en la Universidad de Costa Rica, específicamente deben ser de utilidad real al contexto social, que devuelvan a la sociedad su inversión y no solo se ajusten a un requisito de graduación que no beneficie de manera práctica y real a ningún sector poblacional, correspondiente a la visión y misión de la educación superior pública. En la Escuela de Psicología se han generado un número significativo de manuales $y$ otros materiales, producto de importantes investigaciones que no están siendo utilizados actualmente de manera real y con el potencial necesario. Un ejercicio académico, que pueda dar un impacto positivo y útil a la sociedad es que en futuras investigaciones, se tomen estos materiales y se ajusten a las necesidades reales de los centros, institutos o poblaciones a los que van dirigidos.

\section{BIBLIOGRAFÍA}

Aguilar, I.; Coto, R. y Paniagua, E. "Propuesta metodológica educativa para el manejo nutricional del paciente gastrectomizado del Hospital Max Peralta de Cartago”. [Seminario de graduación para optar por el grado de Licenciatura en Nutrición]. Universidad de Costa Rica, 2001.

Alán, J. y León, M. "Elaboración, aplicación $y$ validación empírica de un modelo de inoculación contra el estrés en pacientes intervenidos neuroquirúrgicamente por lesiones benignas en la columna vertebral en el hospital Dr. R. A. Calderón Guardia”. [Tesis de Licenciatura en Psicología]. Universidad de Costa Rica, 1999.

Alemán, R.; Araya, H.; Soto, P. y Trigueros, J. "Dolor por cáncer. Una aproximación integral del problema". [Tesis de Posgrado en Medicina Familiar y Comunitaria]. Universidad de Costa Rica, 1999.

Alpízar, S. "Determinación de vitaminas liposolubles antioxidantes en suero de pacientes con alto riesgo para cáncer gástrico en Costa Rica”. [Tesis de Magister Scientiae en Ciencias Biomédicas]. Universidad de Costa Rica, 2002. 
Álvarez, M. Stress, un enfoque psiconeuroendocrino. La Habana, Cuba: Editorial científico-técnica, 1989.

Asociación Costarricense de Medicina Forense. "Beneficio al paciente terminal". Medicina Legal 16 (1). Costa Rica: Asociación Costarricense de Medicina Forense. Julio 2000: 18-25.

Asociación Española Contra el Cáncer. Sugerencias para la intervención psicológica en el enfermo oncológico y su familia. Madrid: Asociación Española Contra el cáncer, 1994.

Asociación Española Contra el Cáncer. Informe Anual. Madrid: Asociación Española Contra el cáncer, 2003.

Bandura, A. y Ribes, E. Modificación de la conducta. México: Trillas, 1975.

Baptista, J. "Mitos y realidades acerca del cáncer". [Ponencia presentada en el I Congreso Nacional de Psicooncología]. San José, Costa Rica. Agosto, 2002.

Baréz, M. y Fernández, J. "La inducción de sensación de control como elemento fundamental de la eficacia de las terapias psicológicas en pacientes de cáncer". Revista Anales de Psicología 19 (2). 2003: 235-246.

Barguil, M. "Bienestar psicológico, estrategias de afrontamiento al estrés y autoeficacia percibida en pacientes operados por cáncer gástrico que asisten al Centro de Detección Temprana de Cáncer Gástrico, Hospital Max Peralta, Cartago". [Tesis de Licenciatura en Psicología]. Universidad de Costa Rica, 2003.

Barquero, H. "Cáncer gástrico temprano: experiencia del Centro de Detección Temprana de Cáncer Gástrico entre 1996 y 2002”. [Tesis para optar por el grado de Especialidad de cirugía general]. Universidad de Costa Rica, 2005.

Bayés, R. "Prevención y psicología de la salud". Revista de psicología de la salud 1 (3). 1991: 93-108.

Bayés, R. "Psicología del sufrimiento y de la muerte". Anuario de Psicología 4 (29). 1998: 5-17.

Blanco, M. "Propuesta de un Manual cognitivo conductual emocional para la inoculación del estrés laboral dirigido a gerentes de una empresa privada costarricense". [Tesis para optar por el grado de Licenciatura en Psicología]. Universidad de Costa Rica, 2008.

Bolaños, M. y Rodríguez, G. "Manual de autoayuda cognitivo conductual emocional para el tratamiento del insomnio secundario dirigido a mujeres con cáncer de mama”. [Tesis para optar por el grado de Licenciatura en Psicología]. Universidad de Costa Rica, 2005.

Boyero, B. "Ética de la relación enfermo terminal-entorno familiar". Cuadernos de Bioética 2. 1996: 168-192.

Bowlby, J. La pérdida afectiva. Tristeza y depresión. Barcelona: Paidós, 1984.

Caballo, V. Manual de evaluación y entrenamiento de las habilidades sociales. España: Siglo Xxi Editores SA, 1993.

Calderón, R. y Sancho, A. "Manual cognitivo conductual emocional dirigido a padres de familia de pacientes diagnosticados con retinoblastoma para promover la calidad de vida". [Tesis para optar al grado de Licenciatura en Psicología]. Universidad de Costa Rica, 2005.

Carrobles, J. "Estrés y trastornos psicofisiológicos". En: Caballo, V.; Buela-Casal, G. y Carrobles, J. (dirs.). Manual de psicopatología y trastornos psiquiátricos. Trastornos de la personalidad, medicina conductual y problemas de relación. Vol. 2. Madrid: Siglo XXI Editores SA, 1996.

Cascante, A. y Salazar, C. "Estrés, apoyo social $y$ desempeño académico en estudiantes de $8^{\circ}, 9^{\circ}$ y $10^{\circ}$ nivel de un colegio de San José". [Tesis para optar por el grado de Licenciatura en Psicología]. Universidad de Costa Rica, 1993.

Castro, B. "Alimentos funcionales". Plenitud 7. 2007: 26-27.

Chávez, S. "Repercusiones, reacciones y sentimientos que enfrenta la mujer portadora de lesión ginecológica cancerosa". Revista de Trabajo SocialCCSS 54. 1998: 1078-1090. 
Congreso Nacional de Psicooncología. Memoria del I Congreso Nacional de Psicooncología. San José, Costa Rica: Centro Nacional de Control del Dolor y Cuidados Paliativos, 2002.

Cooper, C. Estrés y cáncer. Madrid: Ediciones Díaz de Santos, 1986.

Córdoba, N. y Peña, L. "Los derechos de los pacientes que sufren enfermedades terminales y los derechos asistenciales de la familia”. [Tesis para optar por el grado de Licenciatura en Derecho]. Universidad de Costa Rica, 2003.

Coto, Y.; Redondo, Y. y Solano, I. "Propuesta de Orientación para facilitar la elaboración del duelo anticipado de los miembros de las familias de los pacientes en fase terminal por cáncer que asisten a la Clínica del Dolor del Hospital William Allen de Turrialba". [Tesis para optar por el grado de Licenciatura en Orientación]. Universidad de Costa Rica, 2001.

Cruzado, J. y Olivares, M. Intervención psicológica en pacientes con cáncer. Madrid: Ediciones Pirámide SA, 1996.

Cuenca, A. y Leiva, K. "Elaboración y validación empírica de un Modelo de Autoayuda Cognitiva Conductual Emocional para el Manejo del Estrés asociado a la Diabetes Mellitus Tipo 2 desde la perspectiva de la Medicina Conductual". [Tesis para optar por el grado de Licenciatura en Psicología]. Universidad de Costa Rica, 2004.

Davis, M.; Robbins, E. y McKay, M. Técnicas de Autocontrol Emocional. Barcelona, España: Editorial Martínez Roca, 1985.

Davidson, J. Asertividad. México: Prentice Hall, 1999.

Deffenbacher, J. "La Inoculación del Estrés". En: Caballo, V. (n.f.). Manual de Técnicas de Terapia y Modificación de Conducta. Madrid, España: Siglo XXI, 1995.

Donker, F. Medicina Conductual y Psicología de la Salud. En: Caballo, V. (n.f.). Manual de Técnicas de Terapia y Modificación de Conducta. Madrid, España: Siglo Veintiuno, 1991.
Donker, F. "Evaluación y tratamiento conductual del dolor crónico". En: Caballo, V. Manual de Técnicas de Terapia y Modificación de Conducta. Madrid, España: Siglo Veintiuno, 1995.

Ellis, A. Humanistic Psycotherapy: the racional-emotive approach. New York: The Julian Press, 1977.

Ellis, A. Una terapia breve más profunda y duradera. Enfoque teórico de la terapia racional emotivo-conductual. España: Paidós, 1998.

Ellis, A. y Abrahmns, E. Terapia Racional Emotiva. México: Alfaomega, 2001.

Ellis, A. y Grieger, R. Manual de terapia racional-emotiva. $3^{\mathrm{a}}$. ed. España: Editorial Desclee de Brouwer SA, 1981.

Fertig, D. "Depression in patients with breast cancer. Prevalence, diagnosis and treatment". The breast Journal 3. 1997: 292-302.

Flikier, B. "Colelitiasis después de gastrectomía radical por cáncer gástrico: experiencia del Centro de Detección Temprana de Cáncer Gástrico”. [Tesis para optar por el título de Médico especialista en cirugía generall. Universidad de Costa Rica, 2006.

Fontana, D. Control del estrés. México: Manual Moderno, 1992.

Folkman, S. y Greer, S. "Promoting psychological well-being in the face of serious illness: when theory, research and practice inform each other". PsychoOncology 9. 2000: 11-19.

González, M. "Jake al Rey: de la psicología patriarcal a la psicología feminista". Revista Costarricense de Psicología 24. 1996: 11-29.

Gonzáles, S. "Un estudio trangeneracional y transcultural de los pensamientos irracionales propuestos por la Terapia Racional Emotiva Conductual”. [Tesis de Licenciatura en Psicología]. Universidad de Costa Rica, 1996.

Gowin, B. y Novak, J. Learning how to Learn. Barcelona, España: Martínez Roca,1988.

Hernández, M. "Sexualidad y cáncer". [Ponencia en I Congreso Nacional de Psicooncología]. Costa Rica: Centro 
Nacional de Control del Dolor y Cuidados Paliativos, 2002.

Hernández, R.; Fernández, C. y Batista, P. Metodología de la Investigación. México: Editorial McGraw-Hill, 1996.

Hernández, R.; Fernández, C. y Batista, P. Metodología de la Investigación. Tercera Edición. México: Editorial McGraw-Hill, 2003.

Jacobson, E. Relajación Progresiva. Chicago: Universidad de Chicago Press, 1929.

Jaramillo, J. El cáncer: fundamentos de Oncología. Tomo 1. Costa Rica: Editorial de la Universidad de Costa Rica, 1991.

Jaramillo, J. Cáncer gástrico: monografía. Costa Rica: Editorial de la Universidad de Costa Rica, 1998.

Kirsta, A. Superar el estrés. Cómo relajarse y vivir positivamente. Barcelona: Integral Ediciones, 1986.

Kraut, A. Los derechos de los pacientes. Buenos Aires, Argentina: Editorial Artes Gráficas Candil, 1997.

Kuble-Ross, E. En caso de muerte y defunción. New York: Mac-Millan, 1969.

Lazarus, R. y Folkman, S. Estrés y procesos cognitivos. Segunda Edición. Barcelona, España: Ediciones Martínez Roca SA, 1986.

Lega, L. y Velten, E. "Albert Ellis". Revista Latinoamericana de Psicología 40 (1). Bogotá, Colombia. Fundación Universitaria Konrad Lorenz, 2008:189-193.

Maguire, P. "Managing psychological morbidity in cancer patients". European Journal of Cancer 36. 2000:556-558.

Martínez, F.; Valiente, J.; Romero, A. et ál. "Dos aproximaciones a la medida de la ansiedad prequirúrgica". Revista de la psicología de la salud 5 (2). 1993: 47-63.

Martínez, F. y Valiente, J. "Marcadores fisiológicos de la ansiedad prequirúrgica”. Cirugía Española 56 (2). 1994: 140144.

McMillan, J. y Schumacher, S. Investigación Educativa. Quinta Edición. Madrid: Person Educación SA, 2005.

Meichenbaum, D. Manual de Inoculación de estrés. Barcelona, España: Ediciones Martínez Roca, 1987.
Méndez, F.; Olivarez, J. y Quiles, M. Técnicas de modificación de conducta. Madrid: Editorial Biblioteca Nueva, 1998.

Mejía, A. y Umaña, V. "El paciente con diabetes Mellitas Tipo II. Plan CognitivoConductual-Emocional para mantener la adherencia a un programa de reducción de peso". [Tesis para optar por el grado de Licenciatura en Psicología]. Universidad de Costa Rica, 2000.

Ministerio de Salud y Organización Panamericana de la Salud. "La OMS se pone en guardia contra la creciente "crisis de sufrimiento'”. Parte I. [Ponencia en Semana Epidemiológica 18]. 27 de abril al 03 de mayo, 1997.

Ministerio de Salud y Organización Panamericana de la Salud. "La OMS se pone en guardia contra la creciente 'crisis de sufrimiento'”. Parte II. [Ponencia en Semana Epidemiológica 19]. 04 de mayo al 10 de mayo, 1997.

Moix, J. "Emoción y cirugía". Anales de Psicología 10 (2). España. Secretariado de Publicaciones e Intercambio científico, Universidad de Murcia, 1994:167175.

Mora, E. "Como enfrentar en familia el impacto que ocasiona una enfermedad crónica". Plenitud 7. 2007: 31-32.

Moreno, M. "Disfrute más su sexualidad". Plenitud 7. 2007: 20-21.

Obando, L. y Villalobos, M. "Procesos de duelo en una familia con un paciente terminal: un acercamiento desde el modelo de Terapia Familiar Sistémico, en una familia costarricense con un miembro varón homosexual con SIDA". [Tesis para optar al grado de Licenciatura en Psicología]. Universidad de Costa Rica, 1994.

Olivares J. y Méndez, F. Técnicas de modificación de la conducta. Madrid, España: Editorial Biblioteca Nueva, 1998.

Peña, L. y Montaña, C. "Manejo del duelo en niños $y$ adolescentes desde el enfoque cognitivo-conductual". En: Caballo, V. Manual de Psicología Clínica Infantil y del Adolescente. Madrid, España: Ediciones Pirámide, 2001. 
Polini, G. "No me cuide, sé lo que necesito". Plenitud 6, 2007: 18-20.

Pourtois, J. y Desmet, H. Epistemología e instrumentación en ciencias humanas. Barcelona: Editorial Herder, 1992.

Ramírez, R. "Perspectiva contemporánea de la modificación de conducta, modelos emergentes". [Ponencia presentada en el XIII Simposio Costarricense de Psicobiología, I Simposio Costarricense de Análisis Conductual Aplicado]. Universidad de Costa Rica, 2003.

Rivas, G. "Participación del personal de enfermería en la atención de las personas que asisten al Centro de Detección Temprana de Cáncer Gástrico en el Hospital Max Peralta en Cartago". [Tesis de Licenciatura en Enfermería]. Universidad de Costa Rica, 2002.

Rojas, S. El manejo del duelo. Una nueva propuesta para un nuevo comienzo. Bogotá, Colombia: Editorial Norma SA, 2008.

Rosch, P. Cáncer y Estrés. Madrid: Alfa Centauro, 1986.

Ruiz, L. y Víquez, R. "Diseño e implementación de un programa de capacitación sobre inoculación de estrés para empleados de una empresa privada costarricense". [Tesis de Licenciatura en Psicología]. Universidad de Costa Rica, 1993.

Russ, B. "Grupos de discusión. De la investigación social a la investigación reflexiva”. En: Galindo, J. Técnicas de Investigación. México: Consejo Nacional para la cultura y las artes, 2002.

Sanz, J. y Modolell, E. "Oncología y Psicología: un modelo de interacción". Psicooncología 1 (1). Madrid: Asociación de Psicooncología (APSOM), 2004: 3-12.

Sierra, F. "Función y sentido de la entrevista cualitativa en investigación social". En: Galindo, J. Técnicas de Investigación. México: Consejo Nacional para la cultura $y$ las artes, 2002.

Sierra, R. "Cáncer gástrico. Epidemiología y prevención". Acta Médica Costa-rricense 44 (2). Costa Rica: Colegio de Médicos y Cirujanos de Costa Rica, 2002: 55-61.
Swartout, H. Consejero Médico del hogar. California: Pacific Press Publishing Association, 1938.

Valverde, G. "El manejo del dolor y los cuidados paliativos: cuestión de actitud, vocación y profesión". Revista de Trabajo SocialCCSS 64. Costa Rica: Caja Costarricense del Seguro Social, Sección de Trabajo Social, 2002: 47-60.

Valladares, R. "Cáncer Gástrico". Normas y procedimientos para el tratamiento del Cáncer en Costa Rica. San José. Costa Rica: Instituto Costarricense contra el Cáncer, 2007.

Vega, W. "Cáncer Gástrico". Normas y procedimientos para el tratamiento del Cáncer en Costa Rica. San José. Costa Rica: Instituto Costarricense contra el Cáncer, 2007.

Vera, M. y Vila, J. "Técnicas de relajación". Caballo, V. (comp.). Manual de técnicas de terapia y modificación de conducta. Madrid: Siglo XXI Editores SA, 1995.

Villalobos, A. "Evaluación de competencias para efectos de selección laboral mediante la Entrevista Conductual". [Tesis de Licenciatura en Psicología]. Universidad de Costa Rica, 2001.

Winawer, S. y Shike, M. Cancer free. Estados Unidos: Simon y Schuster Inc., 1995.

Yanes, J. El control del estrés y el mecanismo del miedo. Madrid: Editorial EDAF, 2008.

\section{REFERENCIAS ELECTRÓNICAS}

Colegio de Médicos y Cirujanos de Costa Rica. Código de Moral y Ética del Colegio de Médicos y Cirujanos de Costa Rica. (n.f.). [Consultado el 20 de Octubre de 2004]. En: <http://colegiodemedicos.sa.cr>

Cortese, A. y Gaynor, E. Las emociones primarias $y$ secundarias. 2005. [Consultado el 30 de marzo de 2009]. En: <http: //www. inteligencia-emocional.org/articulos/ lasemocionesysecundarios.htm>

Ruiz, J. y Cano, J. Manual de psicoterapia cognitiva: la terapia racional emotiva de Ellis. 2008. [Consultado el 09 de marzo de 2009]. En: <http://www.psicologia- 
online.com/ESMUbeda/Libros/Manual/ manual2.htm>

Salaverry, O. "Psicooncología y calidad de vida". Actualidades en psicooncología. 2006. [Consultado el 04 de abril de 2006.]. En: <http://www.angelfire.com/pe/ actualidadpsi/psiconcologia.html>

Universidad Católica de Chile. Cirugía digestiva. 2006. [Consultado el 25 de abril de 2006]. En: <http://escuela.med.puc.cl/ deptos/cxdigestiva/cagastrico.htm>

Velasco, L. "Sexo, género y salud". Educare 21(36). 2006. [Consultado el 26 de setiembre de 2009]. En: <http: // enfermeria21.com/educare/>

PÁGINAS WEB

www.fundacancer.com

www.cancerpancreas.es

www.inteligencia-emocional.org

Fecha de ingreso: 01/07/2010

Fecha de aprobación: 16/03/2011 